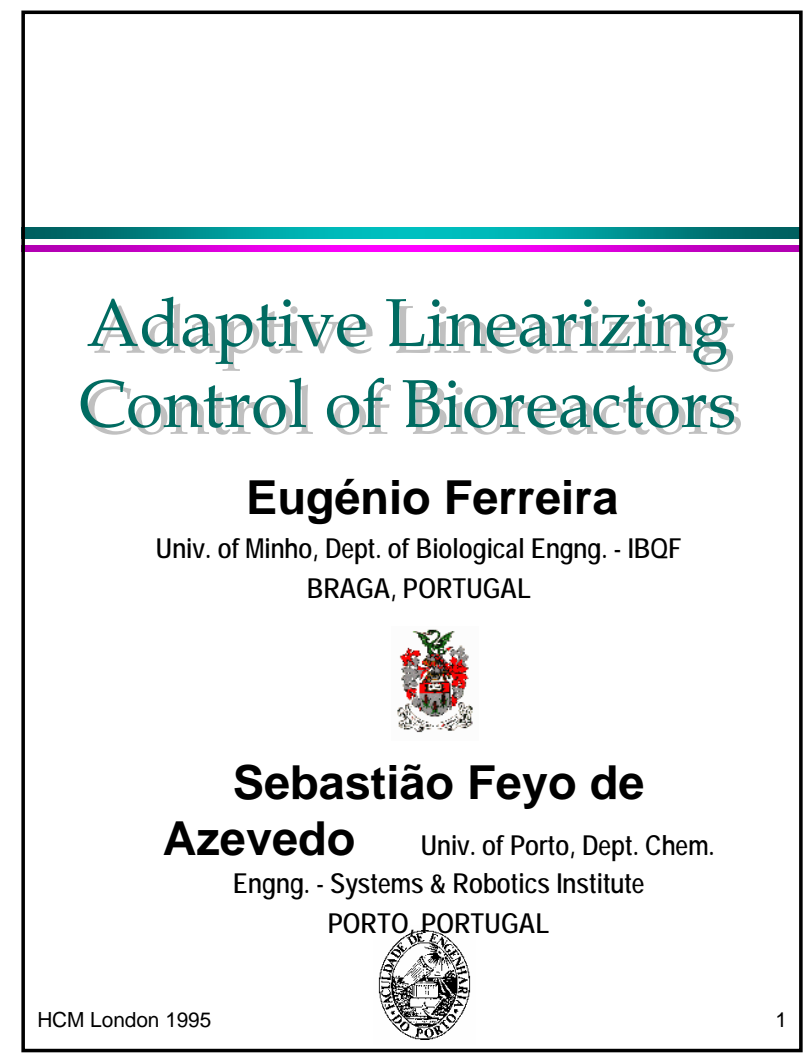

\section{Adaptive Linearizing Control of Bioreactors}

\section{SUMMARY}

Synthesis of SISO and MIMO adaptive linearizing controllers for the regulation of bioreactors.

Case study: Baker's yeast fed-batch fermentation process.

Adaptive feature: on-line estimation of process time-varying parameters.

The adaptive algorithm proposed enforces a desired and pre-set 2nd order convergence dynamics.

\section{Adaptive Linearizing Control}



\section{Linearizing Control Design}

3-step procedure (Bastin \& Dochain):

Step 1: derive a I/O model $\quad \frac{d^{\delta} y}{d t^{\delta}}=f_{0}(t)+u(t) f_{1}(t)$

Step 2: select a stable linear reference model of the tracking error $\left(y^{\star}-y\right)$

$$
\sum_{j=0}^{\delta} \lambda_{\delta-j} \frac{d^{j}}{d t^{j}}\left[y^{*}(t)-y(t)\right]=0
$$

Step 3:Compute the control action such that the I/O model matches the reference model

$$
u(t)=\frac{1}{f_{1}(t)}\left[-f_{o}(t)+\sum_{j=0}^{\delta-1} \lambda_{\delta-j} \frac{d^{j}}{d t^{j}}[y *(t)-y(t)]+\frac{d^{\delta} y *}{d t^{\delta}}\right]
$$


Baker's Yeast Metabolic Pathways

\section{Oxidative Growth on glucose (respiration):}

Glucose + Oxygen $\longrightarrow$ Biomass $+\mathrm{CO}_{2}+$ water

$$
k_{1} S+k_{5} O \stackrel{\mu_{s}^{o}}{\longrightarrow} X+k_{7} C
$$

Redutive Growth on glucose (fermentation):

Glucose

$$
k_{2} S \stackrel{\mu_{s}^{r}}{\longrightarrow} X+k_{8} C+k_{3} E
$$

\section{Oxidative Growth on ethanol (respiration):}

Ethanol + Oxygen $\longrightarrow$ Biomass $+\mathrm{CO}_{2}+$ water

$$
k_{4} E+k_{6} O \stackrel{\mu_{e}^{o}}{\longrightarrow} X+k_{9} C
$$
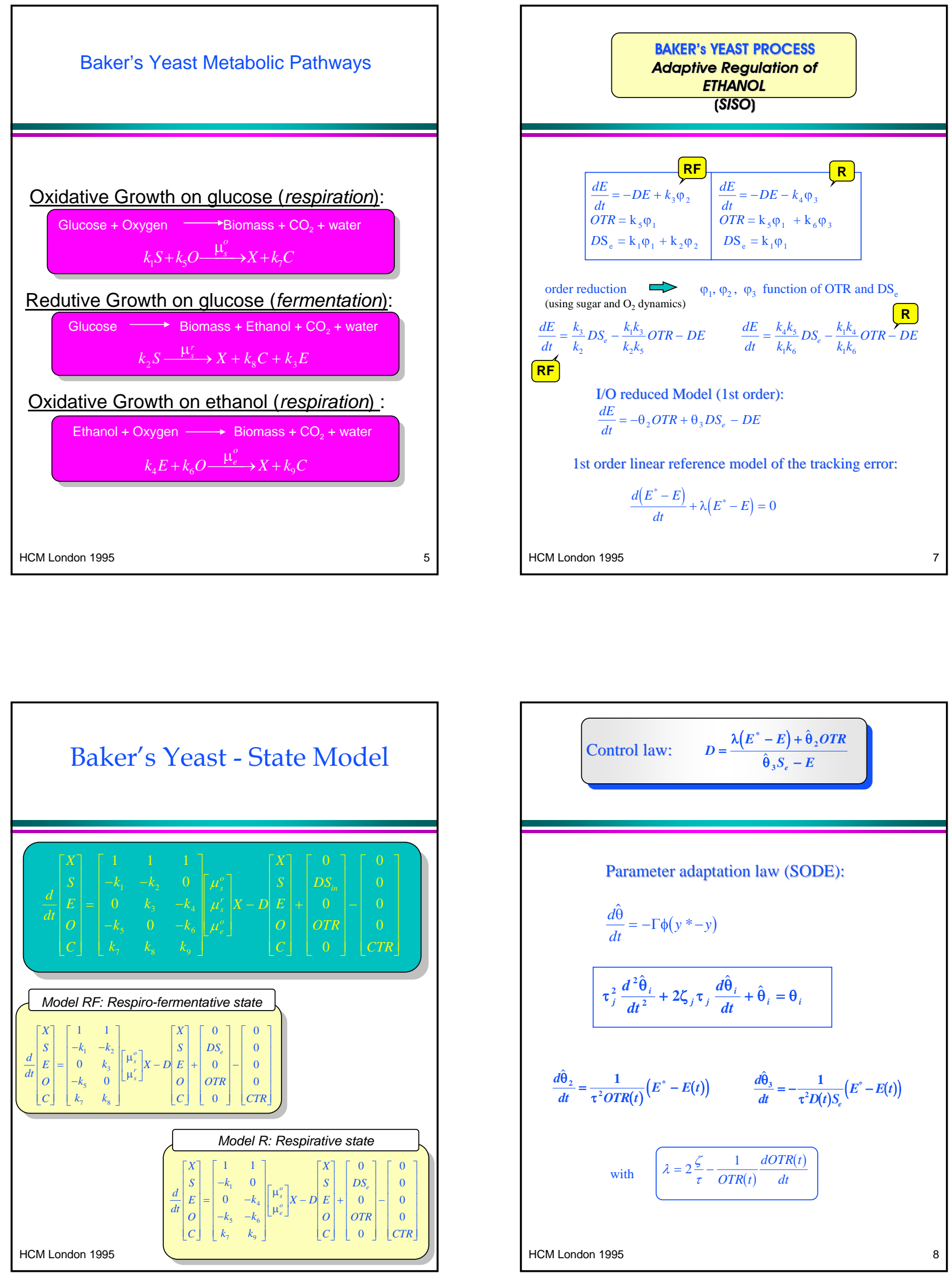


\section{Adaptive Regulation} of ETHANOL and Dissolved OXYGEN (MIMO)

order reduction (using sugar and CO2 dynamics):

$\mathbf{R F} \frac{d}{d t}\left[\begin{array}{l}O \\ E\end{array}\right]=-D\left[\begin{array}{l}O \\ E\end{array}\right]+\left[\begin{array}{cc}-k_{5} & 0 \\ 0 & k_{3}\end{array}\right] \frac{1}{\tilde{k}_{R F}}\left[\begin{array}{cc}-k_{8} & k_{2} \\ k_{7} & -k_{1}\end{array}\right]\left[\begin{array}{c}D S_{e} \\ C T R\end{array}\right]+\left[\begin{array}{c}O T R \\ 0\end{array}\right]$

$\mathbf{R} \int \frac{d}{d t}\left[\begin{array}{l}O \\ E\end{array}\right]=-D\left[\begin{array}{l}O \\ E\end{array}\right]+\left[\begin{array}{cc}-k_{5} & -k_{6} \\ 0 & -k_{4}\end{array}\right] \frac{1}{\widetilde{k}_{R}}\left[\begin{array}{cc}k_{9} & 0 \\ -k_{7} & k_{1}\end{array}\right]\left[\begin{array}{c}D S_{e} \\ C T R\end{array}\right]+\left[\begin{array}{c}O T R \\ 0\end{array}\right]$

generically:

$$
\frac{d}{d t}\left[\begin{array}{l}
O \\
E
\end{array}\right]=-\left[\begin{array}{l}
\theta_{1} \\
\theta_{3}
\end{array}\right] C T R+\left[\begin{array}{l}
1 \\
0
\end{array}\right] \text { OTR }+\left[\begin{array}{c}
\theta_{2} S_{e}-O \\
\theta_{4} S_{e}-E
\end{array}\right] D
$$

Reference model of the tracking error:

$$
\frac{d}{d t}\left[\begin{array}{l}
\left(O^{*}-O\right) \\
\left(E^{*}-E\right)
\end{array}\right]+\left[\begin{array}{ll}
\lambda_{1} & \lambda_{2}
\end{array}\right]\left[\begin{array}{l}
\left(O^{*}-O\right) \\
\left(E^{*}-E\right)
\end{array}\right]=0
$$

Control

Laws:

$$
\left[\begin{array}{c}
\text { OTR } \\
D
\end{array}\right]=\left[\begin{array}{cc}
1 & -\frac{\left(\theta_{2} S_{e}-O\right)}{\left(\theta_{4} S_{e}-E\right)} \\
0 & \frac{1}{\left(\theta_{4} S_{e}-E\right)}
\end{array}\right]\left\{\left[\begin{array}{l}
\lambda_{1}\left(O^{*}-O\right) \\
\lambda_{2}\left(E^{*}-E\right)
\end{array}\right]+\left[\begin{array}{c}
\hat{\theta}_{1} \\
\hat{\theta}_{3}
\end{array}\right] C T R\right\}
$$

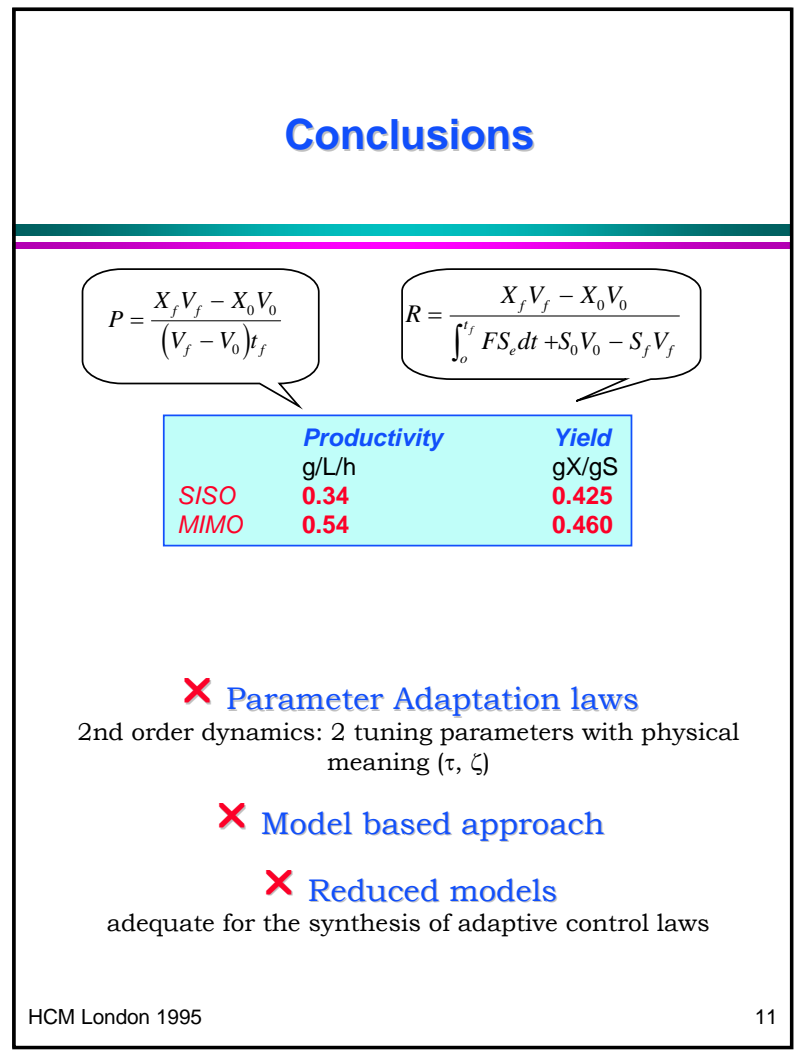

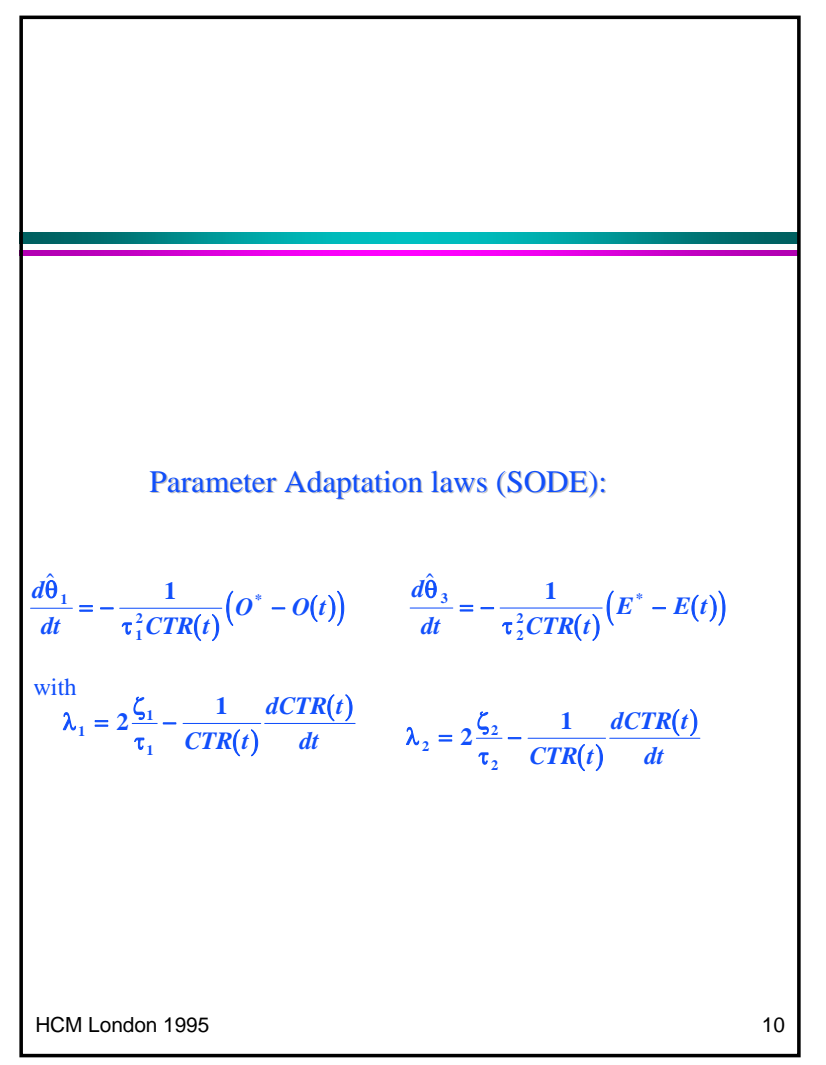

